\title{
An experimental study of the effect of collision properties on spout fluidized bed dynamics
}

Maureen S. van Buijtenen ${ }^{1}$, Matthias Börner ${ }^{2}$, Niels G. Deen ${ }^{1}$, Stefan Heinrich ${ }^{3}$, Sergiy Antonyuk ${ }^{3}$ and J.A.M. Kuipers ${ }^{1}$

${ }^{1}$ Institute for Mechanics Processes and Control Twente, Faculty of Science and Technology, University of Twente, PO Box 217, Enschede, 7500 AE, The Netherlands

${ }^{2}$ Faculty of Process and Systems Engineering, Otto-von-Guericke-University Magdeburg, PO Box 4120, 39106 Magdeburg, Germany

${ }^{3}$ Institute of Solids Process Engineering and Particle Technology, Hamburg University of Technology, 21071 Hamburg, Germany

\begin{abstract}
In this paper we experimentally study the effect of collision properties of different particle systems on the bed dynamics of a spout fluidized bed. This is done in different flow regimes: the spout-fluidization regime (case A), the jet-in-fluidized-bed regime (case B) and the spouting-with-aeration regime (case C). The considered particle systems comprise glass beads, $\gamma$-alumina oxide and zeolite 4A particles, which are all classified as Geldart D. A non-intrusive measurement technique is used, viz. particle image velocimetry (PIV) to measure the particle flow field in a pseudo two-dimensional (2D) spout fluidized bed. Additionally, digital images are analyzed using a newly developed digital image analysis (DIA) algorithm to evaluate the particle volume fraction. It is demonstrated that the new proposed DIA algorithm provides reliable information on the particle volume fraction, showing that it is a powerful tool when combined with PIV. The added value of DIA is confirmed by comparing the particle velocity fields and volumetric particle fluxes.

The particle fluxes display only small differences between the particle systems for each of the examined flow regimes, with the largest difference in the jet-in-fluidized bed regime. In this regime, the flow behaviour of the zeolite 4A particles does not show any features of the spout fluidized bed anymore, but behaviour of an ordinary fluidized bed. This is due to the direct bubble formation above the bottom plate, which results in a large disturbance near the spout entrance. It seems that zeolite 4A particles -because of their small restitution coefficient- tend to cluster in a more pronounced way, producing more bubbles compared to glass beads and $\gamma$-alumina oxide particles.
\end{abstract}

Keywords: Spout fluidized beds, Granulation, Particle Image Velocimetry, Digital Image Analysis

\section{Introduction}

In spout fluidized beds the favourable properties of both spouted and fluidized beds are combined. Spout fluidized beds are frequently used for the preparation of granules or particles through granulation, which are widely applied for example in the production of detergents, pharmaceuticals, food and fertilizers. Due to the high mobility of the particles, undesired agglomeration is prevented and excellent heat transfer control is enabled. During the granulation process, the particles contain different loadings of moisture which results in varying collision properties in time and location across the bed. However, little is known about the effect of the temporal and spatial variation of collision properties on the bed dynamics, and thus on the granulation process. This is mainly due to the fact that spout fluidized beds are not visually accessible. In previous numerical studies (Van Buijtenen et al. 2009 [1]) 
the effect of the collision properties, i.e. the restitution coefficient, on the bed dynamics was investigated. It was found that the influence of the restitution coefficient on the bed dynamics is of paramount importance. To validate these numerical studies, an experimental study is conducted to examine different particle systems with different collision properties in a spout fluidized bed. In this work a non-intrusive measurement technique is used, viz. particle image velocimetry (PIV) to measure the particle flow field in a pseudo two-dimensional (2D) spout fluidized bed. Additionally, digital images are analyzed using a newly developed digital image analysis algorithm (DIA) to determine the local particle volume fraction.

Several workers applied PIV to study 2D granular flows, such as gas-fluidized beds (Bokkers et al. [2], Laverman et al. [3] and Dijkhuizen et al. [4]), spout fluidized beds (Link et al. [5]), spouted beds (Liu et al. [6]), but also vibrated granular beds (Zeilstra et al. [7] and Deng et al. [8]), rotating drums (Jain et al. [9]) and silos or hoppers (Medina et al. [10] and Steingart et al. [11]). Through these applications, PIV has proven to be a powerful tool for 2D granular flow field measurement. However, PIV is generally used to determine velocity profiles of the solids phase rather than individual particle velocities. This means that information about the distribution of the particles inside the bed is lacking. Consequently, Laverman et al. [3] and Link et al. [5] were the first to combine digital image analysis (DIA) with PIV. Laverman et al. [3] used PIV/DIA to correct for the large velocities associated with particle raining through the roofs of the larger bubbles in bubbling fluidized beds. Link et al. [5] used PIV/DIA to determine the particle flux by combining the particle velocity data obtained from PIV with the particle volume fraction gained from DIA, in spout fluidized beds. Hence, these workers show that the combination of DIA with PIV provides much more detailed information about the dynamics of granular systems.

The objective of this work is to study the effect of the collision properties of various particle systems on the bed dynamics in different flow regimes, by employing PIV/DIA to a pseudo-2D spout fluidized bed. The organization of this paper is as follows: first the experimental set-up is briefly discussed. Then, the experimental techniques are described including the newly proposed DIA algorithm, followed by the derivation of the particle volume fraction obtained from DIA. Finally, the particle velocity gained from PIV and the particle flux obtained from PIV/DIA are mutually compared. 


\section{Experimental set-up}

The pseudo-2D spout fluidized bed used in this work is schematically presented in Figure 1. The depth of the bed is assumed to be sufficiently small to display pseudo-2D behaviour and is large enough to avoid extreme particle-wall interaction.

The front wall of the bed consists of a glass plate to enable visual detection of the particle motion. The side walls of the bed are made of aluminium strips and the back wall is made of polycarbonate.

Pressurized air is fed to the bed through three separate sections. The spout section is located in the middle and is surrounded by two sections that provide the background fluidization air. The spout section is covered with a $0.5 \mathrm{~mm}$ gauze and the fluidization section is covered with a $3 \mathrm{~mm}$ thick porous plate with an average pore size of 10 microns. The flow rate of the gas in each section is controlled by mass flow controllers and rapidly responding magnetic valves. Digital images are recorded with a $625 \mathrm{~Hz}$ high speed camera (LaVision Imager Pro) equipped with a $50 \mathrm{~mm}$ lens. The aperture of the camera is set to $\mathrm{f} 6$ and the exposure time is fixed at $0.4 \mathrm{~ms}$. The recorded images consist of $312 \times 1280$ pixels and are stored in the memory of the camera. After all the images for one experiment have been recorded, the 16-bit images are transferred to the hard disk of the PC.

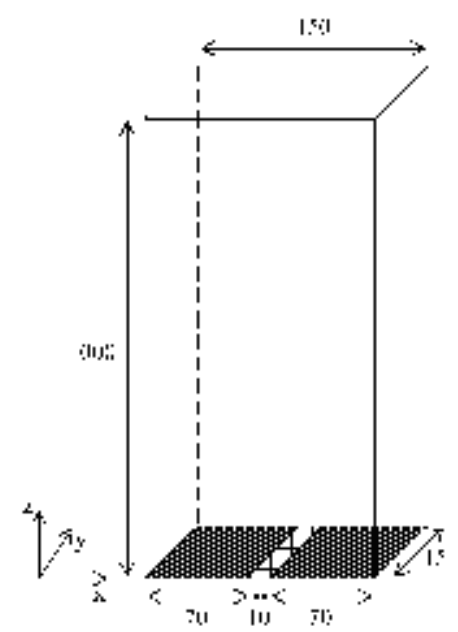

Figure 1 Schematic overview of pseudo-2D spout fluidized bed, with dimensions in mm.

As shown in Figure 2, the pseudo-2D bed is illuminated by two 500W halogen lamps, which are positioned in such a way that the bed is led under a small angle $\left(<45^{\circ}\right)$, preventing undesired reflections. The back wall is covered with a black cloth to provide contrast between particles and gas. The lamps are fed by a direct current supply to minimize temporal variation in the illumination. 
Figure 2 Experimental set-up of pseudo-2D spout fluidized bed, including equipment.

\section{Experimental techniques}

\subsection{Particle Image Velocimetry}

Particle image velocimetry (PIV) is an optical, non-intrusive measurement technique that produces instantaneous 2D velocity data for a whole plane in a 3D flow field. It was originally developed in the field of experimental fluid dynamics to study the flow of continuous fluids (Westerweel et al. [12]). In this work, PIV has been applied to study the particle flow in a pseudo-2D spout fluidized bed. The front view of the bed is recorded using the equipment shown in Figure 2. Two subsequent images of the flow, separated by a short time delay, $\Delta t$, are divided into small interrogation areas. Crosscorrelation analysis is used to determine the volume-averaged displacement, $s_{p}(x, t)$, of the particle images between the interrogation areas in the first and second image. The velocity within the interrogation area is then easily determined by dividing the measured displacement by image magnification, $\mathrm{M}$, and the time delay:

$$
v_{p}(x, t)=\frac{s_{p}(x, t)}{M \Delta t}
$$

provided that $\Delta \mathrm{t}$ is sufficiently small. For further details of this technique, the interested reader is referred to the work of Westerweel at al. [12]. A multi-pass correlation algorithm with a final interrogation size of $32 \mathrm{px}$ was used to obtain the final velocity field. Outliers were removed with a standard median filter. 


\subsection{Digital Image Analysis}

Digital image analysis (DIA) finds its origin in the work of Agarwal et al. [13], where bubbles were detected. Goldschmidt et al. [14] used this technique to detect particles and Link et al. [5] successfully applied DIA to study spout-fluidized beds. In this work, the particles are detected with the use of DIA, and additionally, a new algorithm is proposed to determine the volume fraction of particles in each interrogation area.

The digital image consists of pixels with different intensities, distinguishing the particles from the gas phase. Particles at the first row (in front of the bed) experience the highest intensity and the particles at the back (near by the back wall) have a lower intensity. The lowest intensity is assigned to the gas phase. Due to the fact that homogeneous illumination is difficult to accomplish in practice, the digital image is corrected for inhomogeneities and subsequently the intensities are normalized giving a range between 0 and 1 . The value of 1 represents the brightest particle and 0 the gas phase. Furthermore, the intensities are averaged over interrogation areas with the same size as used for PIV. The averaged intensity represents the apparent 2D volume fraction of the particles. In Figure 3, the original intensity, corrected and normalized intensity and the resulting 2D particle volume fraction are shown.

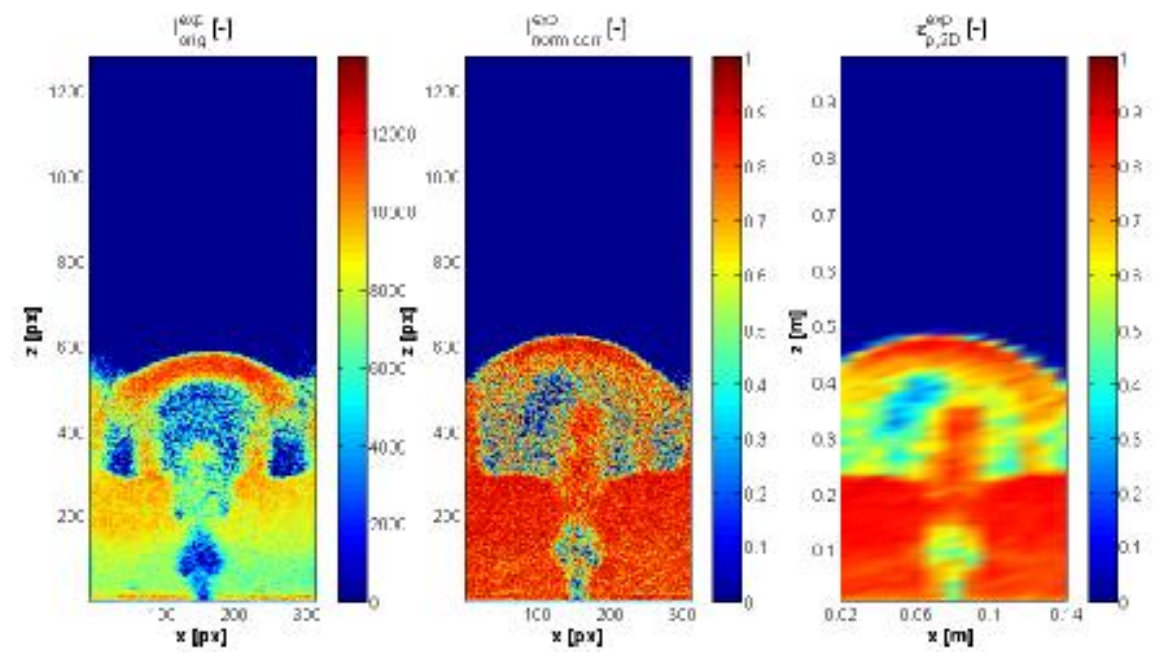

Figure 3 Original intensity, corrected and normalized intensity and resulting 2D particle volume fraction obtained from digital image analysis on experimental image.

In order to translate the apparent 2D particle volume fraction to the true 3D particle volume fraction, a relationship between these two parameters is required. To this end, spout fluidized bed simulation data of a discrete element model (DEM) is used, in which both parameters can be obtained. The 3D particle volume fraction was obtained by applying a porous cube description (see Link et al. [15]). The apparent 2D particle volume fraction was determined from synthetic particle images generated from the particle position data in the DEM simulations. The intensity distribution over a single particle is a Gaussian function and the intensity across the depth of the bed decreases linearly. As a result, each particle obtains the highest intensity in the centre of the particle, and particles in the front of the bed obtain a higher intensity than the particles in the back of the bed. 
Since both the 2D and 3D particle volume fraction are known in the simulation, a relationship is found (by minimizing the summed square of residuals):

$$
\varepsilon_{p, 3 D}=A \cdot \frac{\varepsilon_{p, 2 D}}{\varepsilon_{p, 2 D, \max }-\varepsilon_{p, 2 D}}
$$

with $A=0.0261$ and $\varepsilon_{p, 2 D, \max }=0.837$

This relationship yields linear behaviour for $\varepsilon_{p, 2 D}<<\varepsilon_{p, 2 D, \max }$ and behaves asymptotically for $\varepsilon_{p, 2 D} \uparrow$ $\varepsilon_{p, 2 D, \max \text {. }}$

In Figure 4 the simulation data of 2D and 3D particle volume fraction are plotted, including the resulting fit. In Figure 5, synthetic particle images, 2D and 3D particle volume fraction and the calculated 3D particle volume fraction of the simulations are displayed. The value of the 2D particle volume fraction in the dense regions is difficult to relate to the $3 \mathrm{D}$ particle volume fraction. This is because loosely packed bed regions and densely packed bed regions are difficult to distinguish from each other.

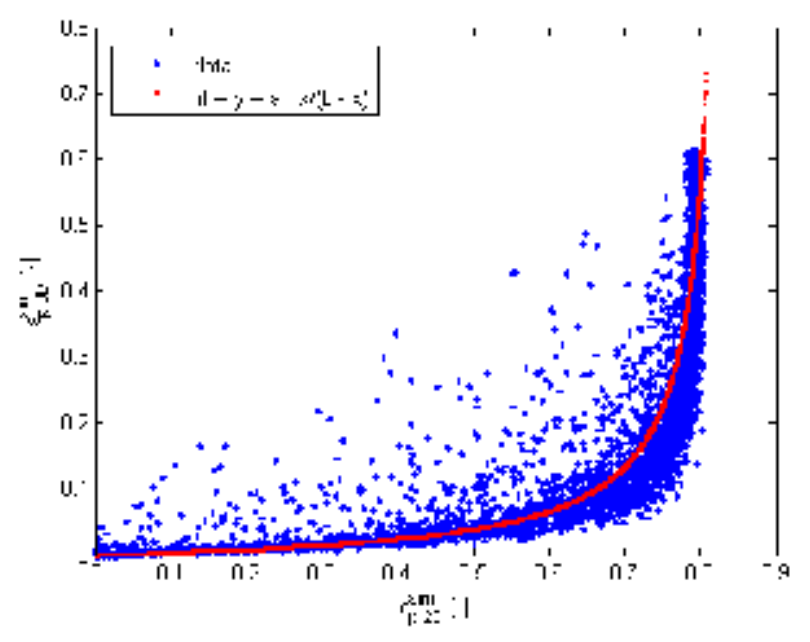

Figure 4 Simulation data of 3D and 2D particle volume fraction including the proposed fit.
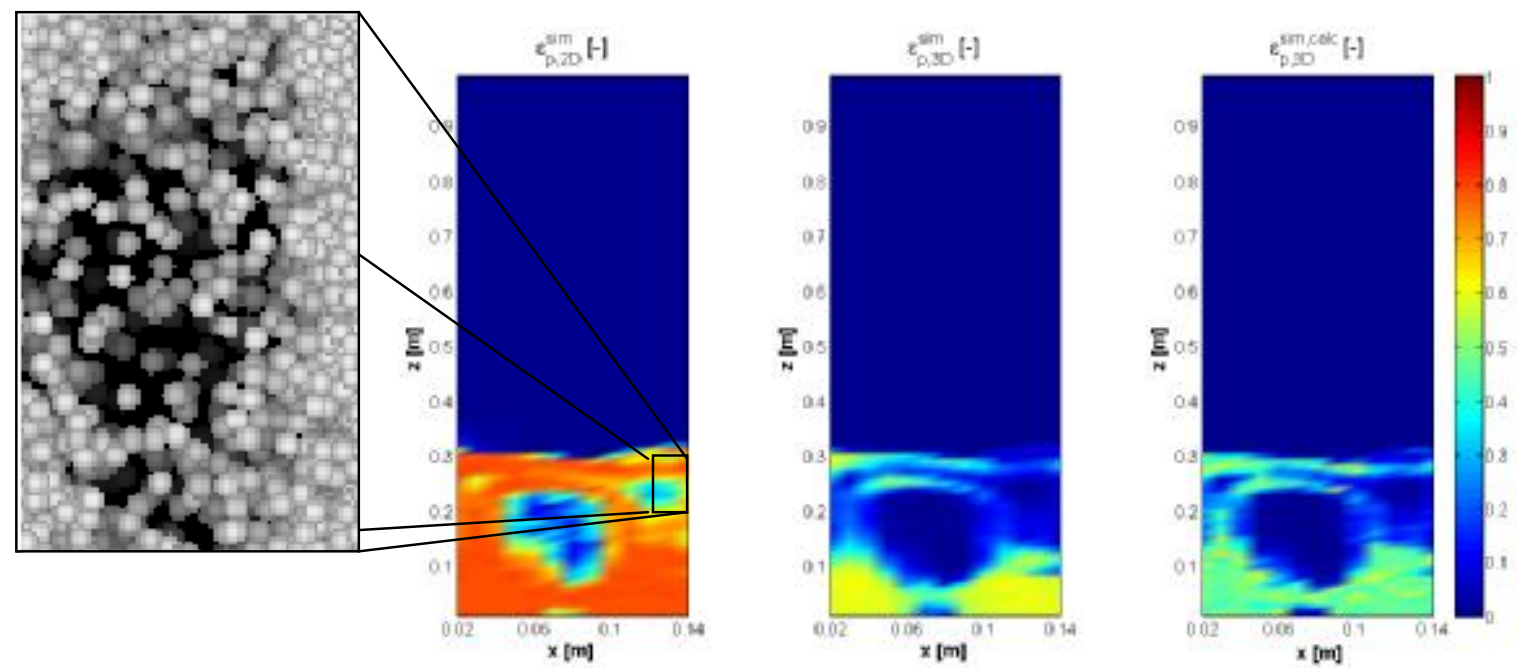

Figure 5 Synthetic generated particle image, 2D particle volume fraction, 3D particle volume fraction and calculated 3D particle volume fraction obtained from digital image analysis on simulated image. 


\section{Test Cases}

The objective of this work is to study the effect of collision properties of Geldart D particles on the bed dynamics. Therefore, three different particle systems, i.e. glass beads, $\gamma$-alumina oxide and zeolite 4A particles are considered. The diameter of the particle systems is chosen such that the particle to bed depth ratio is at least six, to prevent the formation of stable particle structures extending from wall to wall.

Each particle system is studied in three different flow regimes: the spout-fluidization regime (case A), the jet-in-fluidized-bed regime (case B) and the spouting-with-aeration regime (case C). The flow regimes are scaled by the minimum fluidization velocity, to exclude the effect of the particle diameter and density on the bed dynamics. In this way, the collision properties are the only influencing parameters. Tables 1 and 2 respectively summarize the properties of the particle systems and the flow regimes.

Table 1 Properties of particle systems.

\begin{tabular}{|cccc|}
\hline Property & Glass beads & $\gamma$-alumina oxide & Zeolite 4A \\
\hline$d_{p}[\mathrm{~mm}]$ & 1.5 & 1.8 & $1.9-2.3$ \\
$\rho_{p}\left[\mathrm{~kg} / \mathrm{m}^{3}\right]$ & 2526 & 1040 & 1140 \\
$e_{n}[-]$ & 0.87 & 0.74 & 0.65 \\
$u_{m f}[\mathrm{~m} / \mathrm{s}]$ & 0.93 & 0.62 & 0.82 \\
\hline
\end{tabular}

Table 2 Flow regimes.

\begin{tabular}{|cccc|}
\hline Case & Flow regime & $u_{b g} / u_{m f}[-]$ & $u_{s p} / u_{m f}[-]$ \\
\hline A & Spout-fluidization & 1.2 & 23 \\
B & Jet-in-fluidized-bed & 2.3 & 16 \\
C & Spouting-with-aeration & 1.2 & 45 \\
\hline
\end{tabular}

\section{Results and Discussion}

Firstly, the results of the proposed DIA algorithm will be shown, by presenting the time-averaged 3D particle volume fractions. Subsequently, the velocity fields are displayed and compared to the resulting volumetric particle fluxes.

\subsection{Time-averaged 3D particle volume fraction}

The 3D particle volume fraction in the dense packed regions (see Figure 6a) shows some irregularities, especially for cases $\mathrm{A}$ and $\mathrm{C}$. In these regimes, the annulus region is densely packed, since the background velocity is close to the minimum fluidization velocity. Most of the irregularities are caused by the uncertainty in the calibration curve for the dense regions. However, the spots with $\left\langle\varepsilon_{p, 3 D}\right\rangle \approx 0.6$ can be attributed to inhomogeneous illumination that is not fully corrected for. Nevertheless, the timeaveraged 3D particle volume fractions give rather precise information about the distribution of the particles.

For case A (spout fluidization regime) the background velocity is around the minimum fluidization velocity and the spout velocity is high enough to enable the spout channel to form, but not to break through continuously. As a result, the spout channel is periodically blocked causing pressure build up. 
When the pressure is sufficiently high, the spout gas breaks through and launches a cluster of particles higher up in the bed. In Figure $6 \mathrm{~b}$ it is shown that for $\gamma$-alumina and zeolite $4 \mathrm{~A}$ particles, bubble formation in the fountain is more pronounced causing lateral disturbance of the spout channel for zeolite 4A particles. As a result, the periodic ejection of particles is disrupted, which explains the deviation of the time-averaged particle volume fraction for zeolite 4A. For the glass beads and $\gamma-$ alumina particles the spout channel behaves regularly, by which the particle cluster at the top of the fountain is more clearly seen.

For case B (jet-in-fluidized-bed regime), the background velocity is rather high and the spout velocity is moderate. Due to bubble formation in the annulus region, the stability of the spout channel is fully disrupted. Consequently, the typical shape/configuration of a denser packed annulus with a dilute packed spout channel breaks down, as shown in Figure 6b. In contrast to the other particle systems, for zeolite 4A large bubbles are directly formed above the bottom of the bed, causing the smallest dense packed region in the annulus.

For case $\mathrm{C}$ (spouting-with-aeration regime), the spout velocity is very high causing a permanent break through of the spout gas. The spout channel is continuously open and the particles are dragged up high in the bed and subsequently rain down in the annulus region. As shown in Figure 6b, the spout channel for $\gamma$-alumina and zeolite 4A particles still closes in this flow regime. Moreover, for zeolite 4A the closing time of the spout is longer causing laterally disturbance of the spout channel and a higher pressure build up. The zeolite particles are therefore dragged up higher in the bed. Hence, the timeaveraged particle volume fraction displays a lower value in the annulus region compared to the other particle systems, and the spout channel appears to be broader for zeolite $4 \mathrm{~A}$. 

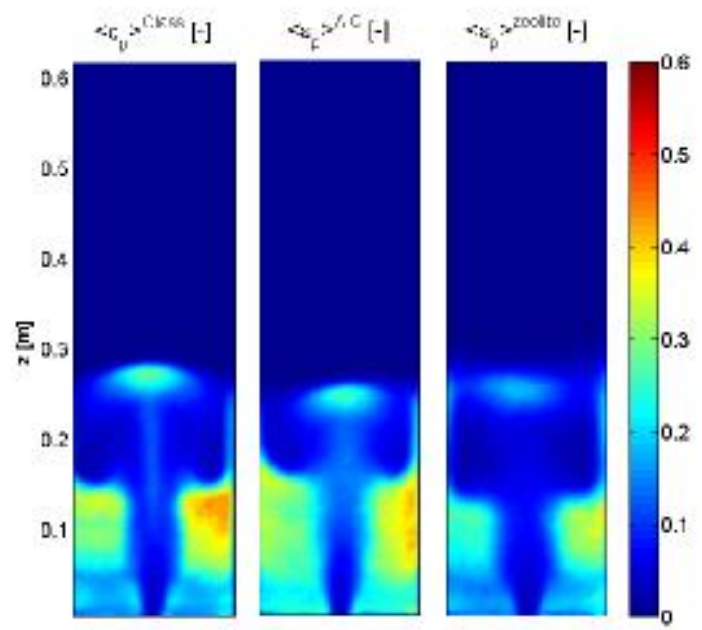

Case A
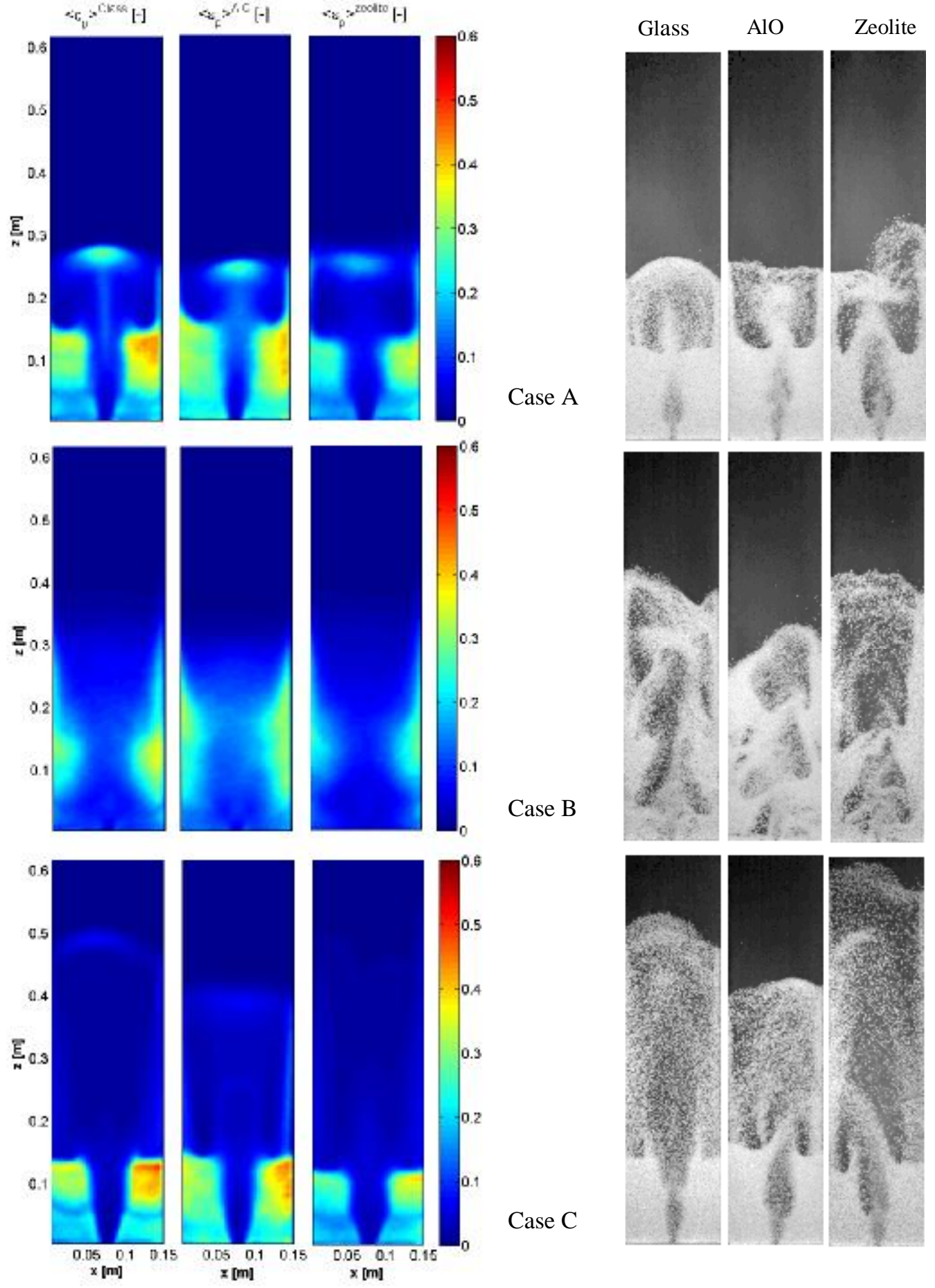

(a)

(b)

Figure 6 Time-averaged 3D particle volume fraction (a) and instantaneous image (b) for spout-fluidization regime (case A), jet-in-fluidized-bed regime (case B) and spouting-with-aeration regime (case C). 


\subsection{Time-averaged particle velocity and volumetric particle flux}

In Figure 7 and 8 the vector fields of the time-averaged particle velocity and time-averaged volumetric particle flux are displayed. The vector field of the time-averaged particle velocity shows the measured velocity of the particles. These graphs, however, give no information about the number of particles that possess that velocity. Viz. the velocity in the spout region is very high, which is only the case for very few particles. This can not directly be inferred from Figure 7. Hence the instantaneous particle velocity is multiplied with the instantaneous particle volume fraction, followed by averaging over time to obtain the time-averaged volumetric particle flux:

$$
<\Phi_{v, p}>=\frac{1}{N_{t}} \sum_{t}^{N_{t}}\left(v_{p}(t) \cdot \varepsilon_{p}(t)\right)
$$

In Figure 8, the time-averaged volumetric particle flux is presented, revealing a clear picture on the motion of the particles. For cases $\mathrm{A}$ and $\mathrm{C}$, the motion of the particles in the annulus region follows the expected behaviour. Although their velocity is very low, the particle flux does give information about the dynamics in the annulus region, because of the high particle volume fraction.

For case A, most differences are observed in the fountain region. For glass beads and $\gamma$-alumina particles, the bubbles in the fountain do not disrupt the motion of the particles, and the direction of the particles at the top of the fountain is therefore vertical. For zeolite 4A particles, however, the direction of the particles is already horizontal at the top of the fountain. This can be attributed to the disturbance caused by the larger bubbles. It seems that for the $\gamma$-alumina particles, the spout gas has more difficulty in breaking through the bed, since its flux is lower compared to the other particles. Consequently, the downward flux in the annulus region is lower as well.

For case B, the flux vector field of zeolite 4A particles displays large resemblance with that of an ordinary fluidized bed, and for glass beads and $\gamma$-alumina particles, the spout fluidized bed pattern is still recognizable. This difference is due to the fact that for zeolite $4 \mathrm{~A}$ particles, large bubbles are directly formed, disrupting the spout channel to such an extent that it looses its influence.

For case C, the patterns depict large similarity for all the particle systems. However, for zeolite 4A more scatter of particles is present at the upper half of the bed. This is due to the laterally disturbance of the spout channel and a higher pressure build up, as mentioned before. Furthermore, the glass beads reach a higher bed height compared to the $\gamma$-alumina particles. This may be due to the difficulty of the spout gas to break through the bed with $\gamma$-alumina, just as in regime A. 

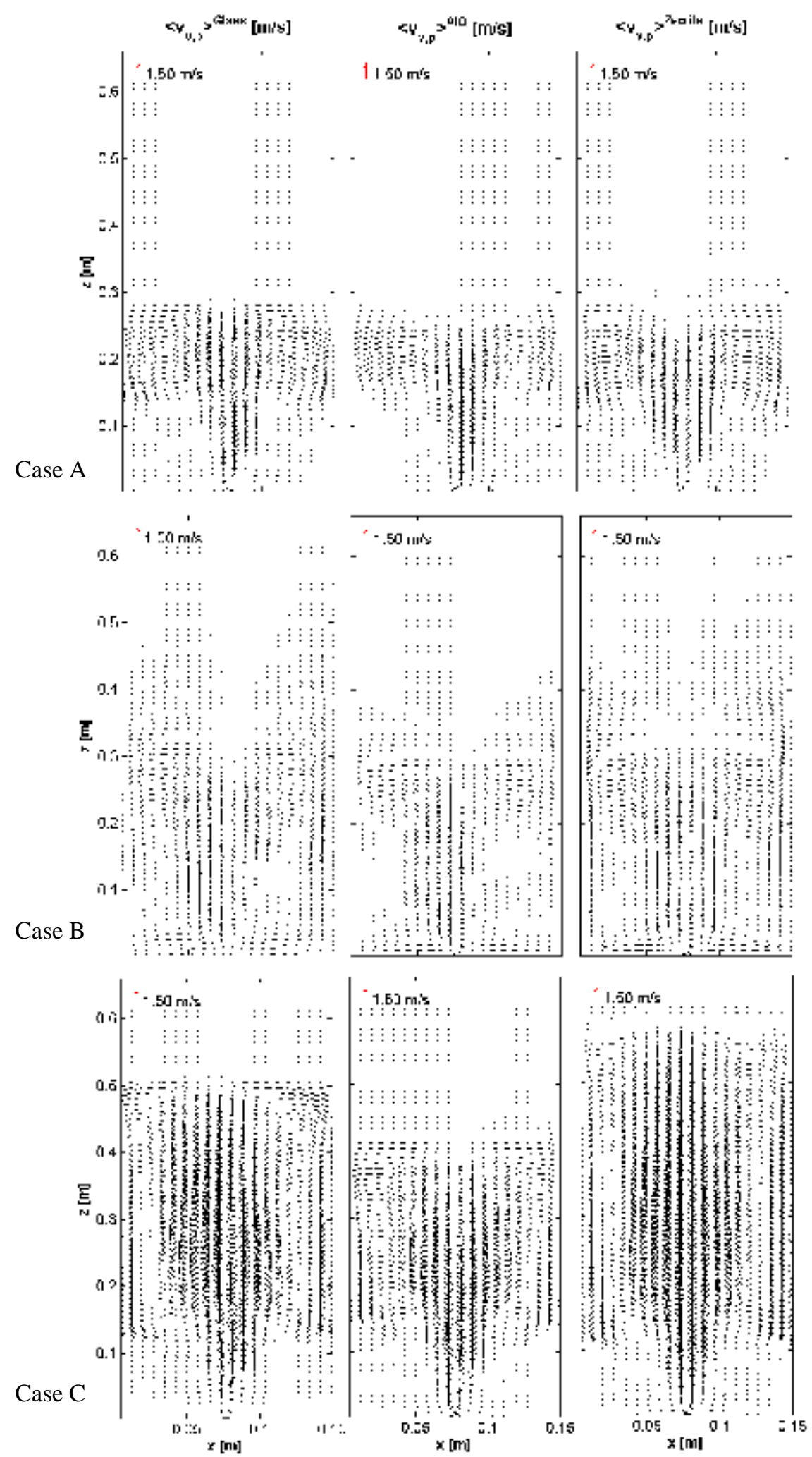

Figure 7 Time-averaged particle velocity for spout-fluidization regime (case A), jet-in-fluidized-bed regime (case B) and spouting-with-aeration regime (case C). Note that only every second vector in each direction is shown for the sake of clarity. 

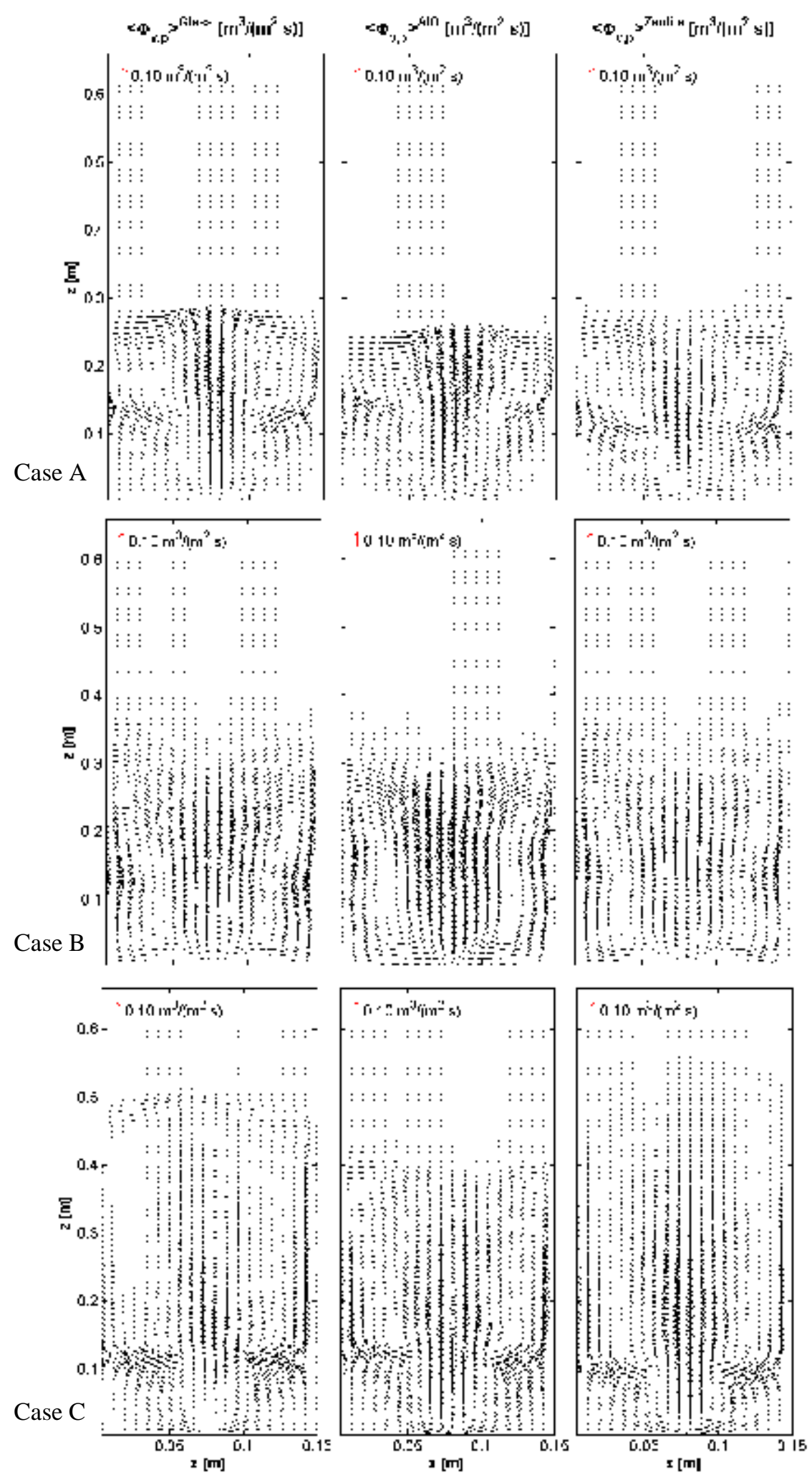

Figure 8 Time-averaged volumetric particle flux $\left[\mathrm{m}^{3} /\left(\mathrm{m}^{2} \cdot \mathrm{s}\right)\right]$ for spout-fluidization regime (case A), jet-in-fluidized-bed regime (case B) and spouting-with-aeration regime (case C). Note that only every second vector in each direction is shown for the sake of clarity. 


\section{Conclusions}

In this work, the effect of the collision properties of different particle systems on the bed dynamics has been experimentally investigated. Particle Image Velocimetry (PIV) and Digital Image Analysis (DIA) were applied to a pseudo-2D spout fluidized bed, to obtain particle velocity fields and the particle volume fraction, non-intrusively. To this end, a new DIA algorithm has been proposed, which successfully translates the apparent $2 \mathrm{D}$ particle volume fraction to the real $3 \mathrm{D}$ particle volume fraction. By employing this DIA algorithm, a more complete picture of the complex solids motion in spoutfluidized beds can be obtained.

The effect of the collision properties (i.e. for. glass beads, $\gamma$-alumina and zeolite 4A particles have been studied in three flow regimes, i.e. the intermediate/spout-fluidization regime (case A), the jet-influidized-bed regime (case B) and the spouting-with-aeration regime (case C). For each flow regime, the particle volume fraction shows small differences between the different particle systems, with the largest deviation for the zeolite $4 \mathrm{~A}$ particles. It seems that the zeolite $4 \mathrm{~A}$ particles tend to cluster in the most pronounced way, due to their smallest restitution coefficient. As a result, more pronounced bubble formation and destabilisation of the spout channel prevails. Additionally, in the spouting-withaeration regime the spout channel closes longer and more frequently. The particles are therefore dragged up higher in the bed leading to a lower particle volume fraction in the annulus region.

The particle fluxes display small differences between the particle systems for each flow regime, with the biggest difference for the jet-in-fluidized bed regime. In this regime, the flow behaviour of the zeolite 4A particles does not show any features of the spout fluidized bed anymore, resembling the behaviour of an ordinary fluidized bed. This is due to the direct bubble formation above the bottom plate, which results in a large disturbance near the spout entrance.

Summarizing, since small differences of the flow behaviour are observed between the particles with different collision properties, it can be concluded that the collision properties only have a small influence on the average bed dynamics. This was mainly shown by the zeolite $4 \mathrm{~A}$ particles, containing the smallest restitution coefficient.

\section{Acknowledgements}

The authors would like to thank FOM, STW and Yara Sluiskil, The Netherlands, for their financial support to the project.

\section{Nomenclature}

$\begin{array}{lll}d & \text { diameter } & {[\mathrm{m}]} \\ e_{n} & \text { coefficient of normal restitution } & {[-]} \\ I & \text { intensity of a pixel } & {[-]} \\ M & \text { image magnification } & {[-]} \\ N & \text { number of time steps } & {[-]} \\ s & \text { volume-averaged displacement } & {[\mathrm{m}]} \\ t & \text { time } & {[\mathrm{s}]} \\ \Delta t & \text { time delay } & {[\mathrm{s}]} \\ u & \text { velocity } & {[\mathrm{m} / \mathrm{s}]} \\ v & \text { velocity } & {[\mathrm{m} / \mathrm{s}]} \\ x & \text { coordinate vector } & {[-]}\end{array}$




\section{Greek letters}

$\varepsilon$

$\rho$

$\Phi$

\section{Subscripts}

corr

$m f \quad$ minimum fluidization

norm normalized

$p \quad$ particle

$t \quad$ time

$v \quad$ volume

\section{Superscripts}

calc

$\exp$

sim volume fraction

density

flux
[-]

$\left[\mathrm{kg} / \mathrm{m}^{3}\right]$

$\left[\mathrm{m}^{3} /\left(\mathrm{m}^{2} \mathrm{~s}\right)\right]$

\section{References}

1. Van Buijtenen, M.S., Deen, N.G., Heinrich, S., Antonyuk, S., Kuipers, J.A.M., 2009. A discrete particle simulation study on the influence of restitution coefficient on spout fluidized bed dynamics. Chemical Engineering and Technology 32, 454-462.

2. Bokkers, G.A., Van Sint Annaland, M, Kuipers, J.A.M., 2004. Mixing and segregation in a bidisperse gas-solid fluidised Bed: A numerical and experimental study. Powder Technology 140, 176-186.

3. Laverman, J.A., Roghair, I., Van Sint Annaland, M., Kuipers, J.A.M., 2008. Investigation into the hydrodynamics of gas-solid fluidized beds using particle image velocimetry coupled with digital image analysis. CJChE 86, 523-535.

4. Dijhuizen W., Bokkers, G.A., Deen, N.G., Van Sint Annaland, M., Kuipers, J.A.M., 2007. Extension of PIV for measuring granular temperature field in dense fluidized beds. AIChE J. 53, $108-118$.

5. Link, J.M., Zeilstra, C, Deen, N.G., Kuipers, J.A.M., 2004. Validation of a discrete particle model in a $2 \mathrm{D}$ spout-fluid bed using non-intrusive optical measuring techniques. CJChE 82, 30-36.

6. Liu, G.Q., Li, S.Q, Zhao, X.L., Yao, Q., 2008. Experimental studies of particle flow dynamics in a two-dimensional spouted bed. Chemical Engineering Science 63, 1331-1141.

7. Zeilstra, C, Collignon, J.G., Van der Hoef, M.A., Deen, N.G., Kuipers, J.A.M., 2008. Experimental and numerical study of wall-induced granular convection. Powder Technology 184, 166-176. 
8. Deng, R., Wang, C.H., 2003. Particle image velocimetry study on the pattern formation in a vertically vibrated granular bed. Physics of Fluids 15, 3718-3729.

9. Jain, N, Ottino, J.M., Lueptow, R.M., 2002. An experimental study of the flowing granular layer in a rotating tumbler. Physics of Fluids 14, 572-582.

10. Medina. A., Córdova, J.A., Luna, E., Treviño, C., 1998. Velocity field measurements in granular gravity flow in a near 2D silo. Physics Letter A 250, 111-116.

11. Steingart, D.A., Evans, J.W., 2005. Measurements of granular flows in two-dimensional hoppers by particle image velocimetry. Part I: experimental method and results. Chemical Engineering Science 60, 1043-1051.

12. Westerweel, J., 1997. Fundamentals of digital particle image velocimetry data. 1997. Meas. Sci. Technol. 8, 1379-1392.

13. Agarwal, P.K., Hull, A.S., Lim, K.S., 1997. Digital image analysis techniques for the tudy of bubbling fluidized beds. In: Chaouki, J., Larachi, F., Dudukovic, M.P. (Eds.), Non-invasive monitoring of multiphase flows, Elsevier Science B.V., Amsterdam, The Netherlands, 407-454.

14. Goldschmidt, M.J.V., Link, J.M., Mellema, S., Kuipers, J.A.M., 2003. Digital image analysis measurements of bed expansion and segregation dynamics in dense gas-fluidised beds. Powder Technology 138, 135-159.

15. Link, J.M., Cuypers, L.A., Deen, N.G., Kuipers, J.A.M., 2005. Flow regimes in a spout-fuid bed: A combined experimental and simulation study. Chemical Engineering Science 60, 3425-3442. 